\title{
FABRICATION OF BIOADHESIVE OCUSERT WITH DIFFERENT POLYMERS: ONCE A DAY DOSE
}

\author{
AYA M. DAWABA ${ }^{1 *}$, HAMDY M. DAWABA ${ }^{2}$, AMAL S. M. ABU EL-ENIN ${ }^{1}$, MAHA K. A. KHALIFA ${ }^{1}$
}

1Department of Pharmaceutics, Faculty of Pharmacy (Girls), Al-Azhar University, Cairo 11651, Egypt. ${ }^{2}$ Department of Pharmaceutics, Faculty of Pharmacy (Boys), Al-Azhar University, Cairo 11651, Egypt

Email: aya.dawaba@yahoo.com

Received: 14 Jul 2018, Revised and Accepted: 29 Oct 2018

\section{ABSTRACT}

Objective: The objective of this current study is to fabricate ocuserts to control the drug release from chosen bioadhesive polymeric matrixes to enhance patient compliance. Ciprofloxacin $\mathrm{HCl}(\mathrm{CFX} \mathrm{HCl})$ was selected as a model drug.

Methods: Different bioadhesive polymers with different film forming capabilities namely Hydroxy Propyl Methyl Cellulose (HPMC K4M), Poly Vinyl Alcohol (PVA), Sodium Carboxy Methyl Cellulose (Na CMC), Hydroxy Propyl Cellulose (HPC), Sodium Alginate (Na Alg.), pullulan and Xanthan Gum (XG) in different ratios were used in fabricating ocuserts using solvent-casting technique. Propylene Glycol (PG) was used as a plasticizer to facilitate the fabrication process. Characterization tests of the developed ocuserts were performed as well as bioadhesive tests and in vitro release studies of the incorporated drug. The obtained results were analysed using different release kinetic models. Stability of the selected ocuserts was investigated at $40 \pm 0.5{ }^{\circ} \mathrm{C}$ and $75 \pm 5 \%$ Relative Humidity (RH) for three months' storage period. In vivo ocular irritation test was performed to investigate the safety of the formula in rabbits' eyes as well as to test the release profile and thus to estimate In vitro In vivo correlation.

Results: All the prepared ocuserts showed the uniformity of film characterization and bioadhesion strength ranged from $240 \pm 66$ and $158 \pm 52$ dyne $/ \mathrm{cm}^{2}$. Selected formula from the in vitro release study tested for in vivo study showed the slow release of ciprofloxacin drug up to $24 \mathrm{~h}$ with no signs of eye irritancy. Results for In vitro In vivo correlation showed an excellent correlation with R² value of 0.9982 .

Conclusion: PVA based ocuserts proven to be a promising once-daily, effective and safe ocular delivery system of the drug.

Keywords: Polymeric matrix, Ocuserts, In vivo tests, Once-daily

(C) 2018 The Authors. Published by Innovare Academic Sciences Pvt Ltd. This is an open access article under the CC BY license (http://creativecommons.org/licenses/by/4.0/) DOI: http://dx.doi.org/10.22159/ijap.2018v10i6.28495

\section{INTRODUCTION}

Eye is an essential organ with unique qualities and enable us to see the world. Drug delivery through ocular route is local more than systemic to bypass the high blood concentration of the drug, which may cause eye damage [1].

The solitary anatomy, biochemistry, and physiology of the eye challenge the formulator to circumvent the protective barriers of the eye without causing any tissue damage because of the impervious nature of the eye [2]. About 70\% of the total ophthalmic preparations available in market are conventional ophthalmic dosage forms (eye drops and ointments). For these dosage forms, frequent dosing is the net result of the availability of just a little amount for its therapeutic effect. That's why to overthrow these issues, newer pharmaceutical technologies such as iontophoresis, nanoparticles, liposomes, nanosuspension, microemulsion, in situ gel and bioadhesive ocuserts have been developed to improve patient compliance by increasing the bioavailability of the drug in a controlled and sustained manner [3-6].

Ocuserts (synonyms of Ocular inserts) are drug delivery devices constructed from polymeric materials with solid or semi-solid consistency, delivering the incorporated drug to the ocular surface by placing it in the conjunctival sac. Ocuserts demonstrate many advantages such as delivering an accurate dose, minimizing systemic drawbacks of ocular remedies, prolong the ocular residence time thus reducing the frequency of administration leading to improving patient compliance, possibility of releasing drugs at a slow and constant rate which from an industry point of view, could increase shelf life stability [7]. Ocular route provides large absorption surface area and high vascularization offering good penetration for hydrophilic, low molecular weight drugs achieving fast onset of action [8]. Compared to oral delivery, the ocular administration provides a potential of dose reduction because it avoids the hepatic first pass metabolism. Therefore, in emergency therapy, ocular administration of convenient drugs would be considered as an alternative to other administration routes [9].
Ocuserts applied behind the eyelid were found to prolong the retention time and precision of dosing [10]. However, films were found to tend to move across the surface of the eye, thus resulting in irritation. It has been shown that the addition of mucoadhesive polymers to ocular films, which can adhere to the epithelial surface [11] reduced film movement across the eye, minimizing ocular irritation and burning sensations [12]. The main disadvantage reported for ocuserts is the annoying sensation accompanied its insertion in the eye, but the numerous advantages of ocuserts supersede this single disadvantage [13] as seen by the implementation of this technology in several successfully marketed ocuserts (Ocusert ${ }^{\circledR}$, Ocufit ${ }^{\circledR}$ SR, and Minidisc ${ }^{\circledR}$ ) [14].

Many ocular regions have poor accessibility to systemic circulation due to the presence of protective barriers which makes the local delivery via topical administration is the favoured route for the treatment of ocular diseases. Typical conditions that require ocular administration include ocular infections and disorders (like conjunctivitis and glaucoma) [2].

$\mathrm{CFX} \mathrm{HCl}$ is a broad-spectrum fluoroquinolone antibiotic that can be taken orally or intravenously [10].

CFX $\mathrm{HCl}$ is used systematically in numerous microbial contaminations such as dermal, pulmonary, urinary tract infections and topically in conjunctivitis and anterior ocular infections [15].

CFX $\mathrm{HCl}$ was the most used antibacterial agent worldwide, and the fifth regularly used generic antibacterial in the USA during the last decade in the $20^{\text {th }}$ century [16].

The aim of the current study was to develop bioadhesive ocuserts for the topical delivery of CFX $\mathrm{HCl}$ suitable for a once a day application employing different bioadhesive polymers.

\section{MATERIALS AND METHODS}

\section{Materials}

$\mathrm{CFX} \mathrm{HCl}$ was a gift sample from Eipico, Egypt. Pullulan was purchased from VWR International LLC, West Chester, PA, USA. HPC, HPMC K4M, 
PG, and Glycerin were obtained from ADWIC, El-Nasr Chemical Co., Cairo, Egypt. Na Alg., Di-Sodium Hydrogen Phosphate $\left(\mathrm{Na}_{2} \mathrm{HPO}_{4}\right)$ and Potassium Di-Hydrogen Phosphate $\left(\mathrm{KH}_{2} \mathrm{PO}_{4}\right)$ were supplied from ElNasr pharmaceuticals company, Cairo, Egypt. Na CMC and PVA were supplied from Sigma Company for pharmaceuticals, Cairo, Egypt and were used without further purification.

\section{Methods}

\section{UV scanning solution preparation}

A stock solution of $\mathrm{CFX} \mathrm{HCl}$ was prepared by placing accurately weighed $100 \mathrm{mg}$ in a $100 \mathrm{ml}$ measuring flask and freshly prepared phosphate buffer $\mathrm{pH}$ 7.4was added till reaching the mark. From the resultant solution, $1 \mathrm{ml}$ was taken and completed to $100 \mathrm{ml}$ with phosphate buffer $\mathrm{pH} 7.4$ and then scanned in the $200-400 \mathrm{~nm}$ using a UV-spectrophotometer (Shimadzu UV spectrophotometer, 1601PC double-beam spectrometer, Kyoto, Japan).

\section{FTIR spectroscopy}

Drug identification and detection of any possible incompatibility existed between the excipients is done through Fourier transform infrared (FTIR) spectroscopy. CFX HCl powder and the formula (F-I) blend were examined using FTIR spectrophotometer (model Impact 410, Milwaukee, WI, USA) with a scanning range 500 to $4000 \mathrm{~cm}^{-1}$ and the resolution was $1 \mathrm{~cm}^{-1}$. $\mathrm{KBr}$ disk method was used to obtain the FTIR spectra of $\mathrm{CFX} \mathrm{HCl}$ and the formulation (F-I) blend. The infrared peaks of pure $\mathrm{CFX} \mathrm{HCl}$ were analysed and compared with the peaks obtained for the formulation (F-I) blend to identify if there is the deletion of or additional formation of peaks.

\section{Fabrication of ocuserts}

CFX $\mathrm{HCl}$ ocuserts were prepared by solvent casting technique using HPMC K4M, pullulan, CMC, HPC, Na Alg., PVA and XG as biodegradable bioadhesive polymers $[17,18]$. All polymers were completely dissolved in distilled water (left overnight for uniform dispersion) except PVA which was dissolved in hot water at $70{ }^{\circ} \mathrm{C}$. After dissolving the polymers in water, $\mathrm{PG}$ as a plasticizer was added (2.5\% w/w of polymer). Bath sonicator (Model SS101H 230, Sonix IV, CA, USA) was used to remove air bubble appeared after addition of $\mathrm{CFX} \mathrm{HCl}$ into the polymeric solution. After complete mixing of drug and polymer, $10 \mathrm{ml}$ of the clear solution was poured into the clean petridish moistened with glycerin. The petri dish was covered with an inverted glass funnel of stem orifice $0.6 \mathrm{~cm}$ in diameter with a cotton plug closing the stem of the funnel. Clearance was provided for the escape of the solvent vapors by raising the base of the funnel $(2 \mathrm{~cm})$ just above the resting surface. The funnel was an aid to control the rate of evaporation of the solvent and reducing the blistering of the surface of the deposited film [19]. After complete evaporation of the solvent, cast films were obtained, cut into definite circular pieces by cork borer $(8 \mathrm{~mm})$, wrapped in an aluminum foil and stored in $\mathrm{CaCl}_{2}$ desiccator at room temperature in a dark place for further evaluation studies.

Table 1: Formulation of ocuserts with the different film-forming agents*

\begin{tabular}{|c|c|c|c|c|c|c|c|}
\hline Formula code & HPMC K4M & PVA & CMC & HPC & Na Alg. & Pullulan & $\mathbf{X G}$ \\
\hline F-I & $1.5 \%$ & & & & & & \\
\hline F-II & & $1.5 \%$ & & & & & \\
\hline F-III & & & $1.5 \%$ & & & & \\
\hline F-IV & & & & $1.5 \%$ & & & \\
\hline F-V & & & & & $1.5 \%$ & & \\
\hline F-VI & & & & & & $2 \%$ & \\
\hline F-VII & & & & & & & $1.5 \%$ \\
\hline
\end{tabular}

*All formulae were prepared with 2.5\% PG as a plasticizer and $17 \mathrm{mg}$ of $\mathrm{CFX} \mathrm{HCl}$.

\section{Characterization of film fabrication}

The thickness of the ocusert was measured using a film thickness tester (Vernier caliper, Shanghai, China) at various regions of the film $(n=3)[20,21]$.

Three films of the same size were weighed on an electronic digital balance (Mettler AJ 100, Switzerland). The average weight, as well as the weight variation, were calculated [20-22].

Petri dish containing $0.5 \mathrm{ml}$ distilled water was used to moisten the ocusert for $30 \mathrm{~s}$. Then the $\mathrm{pH}$ meter electrodes (Digital pH meter, Toshniwal Pvt. Ltd., India) were brought in contact with the surface of the ocusert and left for 1 min for equilibration. The $\mathrm{pH}$ value was noted and recorded [20,21].

To determine moisture loss, weighted ocusert was placed in a desiccator containing anhydrous calcium chloride. After three days the film was reweighed, and moisture loss was calculated using the following equation [23, 24].

$$
\text { Moisture loss }(\%)=\left(\mathrm{W}_{\mathrm{i}}-\mathrm{W}_{\mathrm{f}} / \mathrm{W}_{\mathrm{i}}\right) \times 100 \ldots
$$

Where $\mathrm{W}_{\mathrm{i}}$ is the initial weight, and $\mathrm{W}_{\mathrm{f}}$ is the final weight of the film. The study was done in triplicate for each ocusert formulation.

For Moisture gain study, weighted ocusert was placed in a desiccator containing $100 \mathrm{ml}$ of saturated solution of aluminum chloride to maintain $80 \%$ humidity. After three days film was reweighed, and moisture gain was calculated using the following equation [23, 24].

$$
\text { Moisture gain }(\%)=\left(\mathrm{W}_{\mathrm{f}}-\mathrm{W}_{\mathrm{i}} / \mathrm{W}_{\mathrm{i}}\right) \times 100 \ldots . .(2)
$$

Where $\mathrm{W}_{\mathrm{i}}$ is the initial weight, and $\mathrm{W}_{\mathrm{f}}$ is the final weight of the film. The study was done in triplicate for each ocusert formulation.
Folding endurance was determined manually in triplicate by repeatedly folding an ocusert at the same place by using forceps till broken. The numbers of folding the ocusert without being broken were calculated, and the standard deviation (SD) was estimated [24].

Drug content was analysed by dispersing the ocusert in $20 \mathrm{ml}$ of freshly prepared phosphate buffer $\mathrm{pH}$ 7.4. The solution was stirred and filtered through Whatman filter paper no 1 . About $1 \mathrm{ml}$ solution was withdrawn, suitably diluted with phosphate buffer $\mathrm{pH} 7.4$ and the drug content in the solution was measured by a UV-Visible spectrophotometer (Shimadzu UV spectrophotometer, 1601-PC double-beam spectrometer, Kyoto, Japan) at $\lambda_{\max } 278 \mathrm{~nm}[23,24]$.

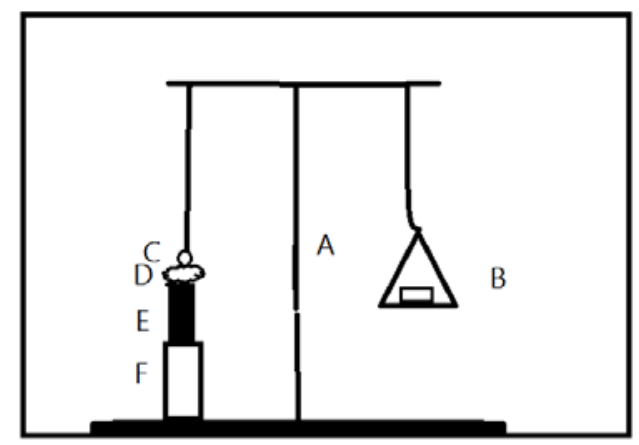

Fig. 1: Schematic diagram of the device used for bioadhesion test (A) modified-balance (B) weight (C) rubber stopper (D) cornea with ocusert (E) glass vial (F) support to the vial to adjust weight 


\section{Bioadhesion characterization}

\section{Ex vivo bioadhesive strength}

The freshly excised cornea of a cow was used as a model mucous membrane for the measurement of bioadhesive strength. Fresh cow cornea was obtained from a local slaughterhouse and used within $2 \mathrm{~h}$ of slaughter. The mucosal membrane was separated by removing the underlying fat and loose tissues. The membrane was washed with distilled water and then with isotonic phosphate buffer $\mathrm{pH} 7.4$ at $37^{\circ} \mathrm{C}$.

The bioadhesive strength of ocusert $(n=3)$ was measured on a modified two-arm physical balance as illustrated in fig. $1[25,26]$.

The pan at the left arm of the balance was detached, and a vertical thread was tied to its end. A rubber stopper, hanging downward, was hung to the lever of the left arm. The ocusert to be tested adhered to the downward facing side of the rubber stopper. Cow cornea was tied onto the open mouth of a glass vial filled with isotonic phosphate buffer. The vial was fitted in the centre of a glass beaker fill illustrated ed with simulated tear fluid (STF) with $\mathrm{pH} 7.4,37^{\circ} \mathrm{C} \pm 1^{\circ} \mathrm{C}$. The apparatus was set such that the vial (mucosal membrane tied on it, facing upward) lies exactly below the rubber stopper (patch adhered onto it, facing downward). The rubber stopper was lowered so as to make the ocusert come in contact with the membrane. After facilitating the contact between the two, weight was put on the right limb of balance and increased gradually until the ocusert got detached from the cornea. The weight (gram force) required to detach the ocusert from the mucosal surface gave the measure of detachment stress, calculated by:

$$
\text { Detachment stress }\left(\frac{\text { dyne }}{\mathrm{cm} 2}\right)=\frac{W \times g}{A} \ldots \ldots \ldots . . .(3)
$$

where $\mathrm{w}$ is the weight required for the detachment of ocusert, $\mathrm{g}$ is the acceleration due to gravity considered as $980 \mathrm{~cm} / \mathrm{s}$, and A is the area of the mucosal surface exposed $\left(\mathrm{cm}^{2}\right)$ [25-27].

\section{Ex vivo bioadhesion time}

The ex vivo bioadhesion time was ascertained $(n=3)$ after application of the ocusert onto freshly cut cow cornea. The fresh cornea was fixed in the inner side of the beaker, above $2.5 \mathrm{~cm}$ from the bottom, with cyanoacrylate glue. One side of each ocusert was wetted with one drop of isotonic phosphate buffer $\mathrm{pH} 7.4$ and pasted to the cornea by applying a light force with a fingertip for $30 \mathrm{~s}$. The beaker was filled with $500 \mathrm{ml}$ of STF pH 7.4 and was kept at $37^{\circ} \mathrm{C} \pm 1$. Ocusert adhesion was monitored up to $6 \mathrm{~h}$ and the time required for the ocusert to detach from the cornea was recorded as the mucoadhesion time [25-27].

\section{In vitro drug release study}

The in vitro drug diffusion from the ocuserts was studied using the cylindrical glass tube (Internal diameter $15 \mathrm{~mm}$ and length $100 \mathrm{~mm}$ ). The diffusion cell membrane (Prehydrated cellophane membrane) was tied to one end of the cylindrical tube, which acted as a donor compartment and the ocusert was placed inside this compartment. The entire surface of the membrane was in contact with $25 \mathrm{ml}$ isotonic phosphate buffer ( $\mathrm{pH}$ 7.4) placed in a $50 \mathrm{ml}$ beaker (receptor compartment). Shaking water bath (FALC Model WB-MF, FALC instrument, Italy) was used to shake the contents of the receptor compartment continuously at constant temperature $\left(37 \pm 0.5{ }^{\circ} \mathrm{C}\right)$. At definite time intervals $(1 \mathrm{~h}), 1 \mathrm{ml}$ of the release solution was withdrawn from the receptor compartment and replaced with freshly prepared phosphate buffer ( $\mathrm{pH}$ 7.4). The aliquot solution was analysed for the drug content using UV-Visible spectrophotometer (Shimadzu UV spectrophotometer, 1601-PC double-beam spectrometer, Kyoto, Japan) at $\lambda \max 278 \mathrm{~nm}[23,24]$.

\section{Drug release kinetic study}

To determine the exact mechanism of drug release from the ocuserts, the in vitro drug release data obtained was analysed using Zero order, First order and Higuchi square root equation [28, 29].

\section{Stability study}

Accelerated stability studies might serve as a tool for formulation screening and stability issues related to shipping or storage at room temperature [30].
The accelerated stability studies were carried out in accordance with the ICH guidelines [31]. Enough ocuserts (packed in aluminium foil) were stored, with RH of $75 \%$ and at a temperature of $40 \pm 0.5^{\circ} \mathrm{C}$ for three months. The samples were tested for drug content after 0,7 , 15,30 and 90 d respectively.

\section{In vivo ocular irritation test}

Approval for the use of animals in the study was obtained from the Al-Azhar University, Faculty of Pharmacy, Animal Ethics Committee (Ref. No.166/2018) which comply with the 3ARRIVE guidelines. The current experiment was carried out in accordance with the U. K. Animals (Scientific Procedures) Act, 1986 and associated guidelines, EU Directive 2010/63/EU for animal experiments. In addition, all institutional and national guidelines for the care and use of laboratory animals were followed.

Our in vivo study aims to determine the objective irritation potential of the prepared ocusert and the in vivo drug profile of the selected ocusert. The rabbit was chosen as a model for this study because its eye simulates an adult human eye with respect to size, shape, physiology, and composition of tears (32).

New Zealand rabbits of either sex weighing 2.5 to $3.5 \mathrm{~kg}$ were used to measure the in vivo ocular irritation test in the eye. The rabbits were purchased from the Nile Company for pharmaceuticals and chemical industries (Al Sawah St, Cairo, Egypt).

The animals were housed in cages in the animal house (located in the faculty of pharmacy (boys branch), Al-Azhar university, Cairo, Egypt) under controlled conditions of temperature $(27 \pm 2 \mathrm{C})$ and light. They were fed with standard laboratory diet, and water was provided ad libitum. Ethical clearance for the handling of experimental animals was obtained. The rabbits were fed balanced diet pellets and maintained in a temperature-controlled room, at 20 ${ }^{\circ} \mathrm{C}$ to $24{ }^{\circ} \mathrm{C}$ before the experiment. 6 animals were used in the experiment where free leg and eye movement was allowed. The investigated ocusert was placed in the left eye while the right eye of each rabbit was considered the control. The ocusert was sterilized by using UV radiation before in vivo study. The ocuserts and other materials were exposed to UV radiation for $1 \mathrm{~h}$ [33]. After sterilization, ocusert was transferred into polyethylene bag with the help of forceps inside the sterilization chamber itself. The observations based on scoring approach $(0=$ normal; $3=$ worst $)$ according to Peyman scale. [34] Peyman et al., [34] established the safety of the developed ocuserts in the rabbit eye. Ocusert was removed each hour for the first eight hours to determine the amount of drug released and hence evaluate in vitro in vivo correlation. The amount of drug remaining in each ocusert was determined as per the assay method of the drug in ocuserts given in drug content. Cumulative percent drug released (CDR) in vivo was calculated [35, 36]

\section{Sterility test}

Any preparation intended to be placed in the eye must be sterile, therefore, testing the sterility is a very important evaluation parameter. The tests for sterility were done by detecting the presence of viable forms of bacteria, fungi, and yeast in or on preparations. The tests were carried out under strict aseptic techniques to avoid accidental contamination of the preparation [37].

The sterility test was performed according to the guidelines of Indian Pharmacopoeia applying direct inoculation method. $2 \mathrm{ml}$ of prepared CFX $\mathrm{HCl}$ ocusert solution was removed with a sterile needle and aseptically transferred to Medium A (fluid thioglycollate medium) and Medium B (soyabean-casein digest medium) separately. After mixing with each medium, incubation of each medium for $7 \mathrm{~d}$ was done (The incubation temperature was $30^{\circ} \mathrm{C}$ to $35^{\circ} \mathrm{C}$ in the case of fluid thioglycolate medium and $20^{\circ} \mathrm{C}$ to $25^{\circ} \mathrm{C}$ in the case of soyabean-casein digest medium) [37].

\section{Microbiological studies}

Staphylococcus aureus (S. aureus; ATCC ${ }^{\circledR} 25923$ ) test microorganism purchased from American Type Culture Collection (PO Box 1549, Manassas, VA 20108 USA) was used to assess the biological activity of the selected ocusert formulation. The test organism was seeded in 
nutrient agar and allowed to solidify in the petri dish. An ocusert was carefully placed over the agar layer at a suitable distance [38]. The plates were then incubated at $37 \pm 0.5{ }^{\circ} \mathrm{C}$ for $24 \mathrm{~h}$. After incubation, the obtained zone of inhibition was compared with the control ocusert (i.e. ocusert with no drug).

\section{RESULTS AND DISCUSSION}

\section{UV scanning}

The stock solution was scanned in the 200-400 nm UV regions. The wavelength adopted for absorbance measurement was the observed wavelength maximum $\left(\lambda_{\max }\right)$ at $278 \mathrm{~nm}$.

\section{FTIR spectroscopy}

The characteristic absorption bands of CFX $\mathrm{HCl}$ at 1281.59 and 1612 $\mathrm{cm}^{-1}$ (fig. 2b) was due to the stretching vibration of C-F bond and the vibration of phenyl framework conjugated to- $\mathrm{COOH}$, respectively. The stretching vibration at $1705.07 \mathrm{~cm}^{-1}$ was due to $-\mathrm{COOH}$ and the stretching vibrations of $\mathrm{C}-\mathrm{H}$ from the phenyl framework at 2962.66 and $2908.65 \mathrm{~cm}^{-1}$ were also observed. All the peaks were also found in drug-loaded ocusert that confirms the presence of the drug in the polymers without any interaction.

\section{Fabrication of ocuserts}

Ocuserts were prepared using polymers pullulan, HPC, HPMC K4M, CMC, Na Alg., PVA and XG. The selection of polymers depended on their film-forming properties, their biodegradability and retardant to biodegradability to provide sustained release pattern. As shown in fig. 3, the prepared ocuserts were found to be satisfactory uniform, transparent and flexible [39].

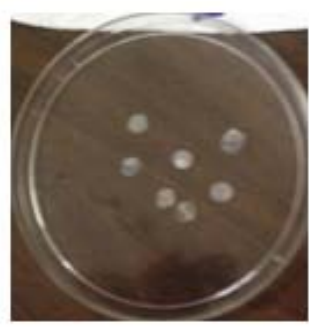

I

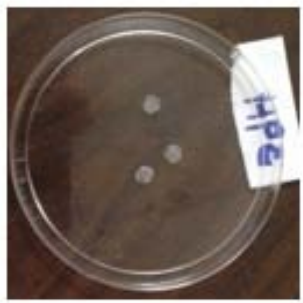

IV

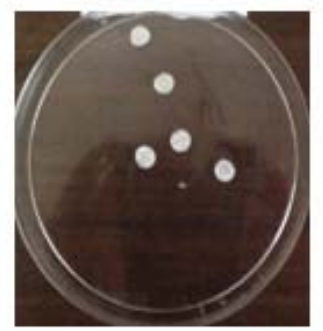

II

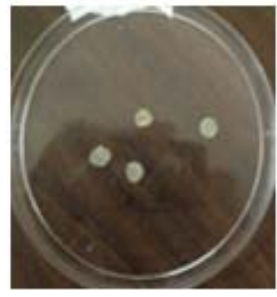

$\mathrm{V}$

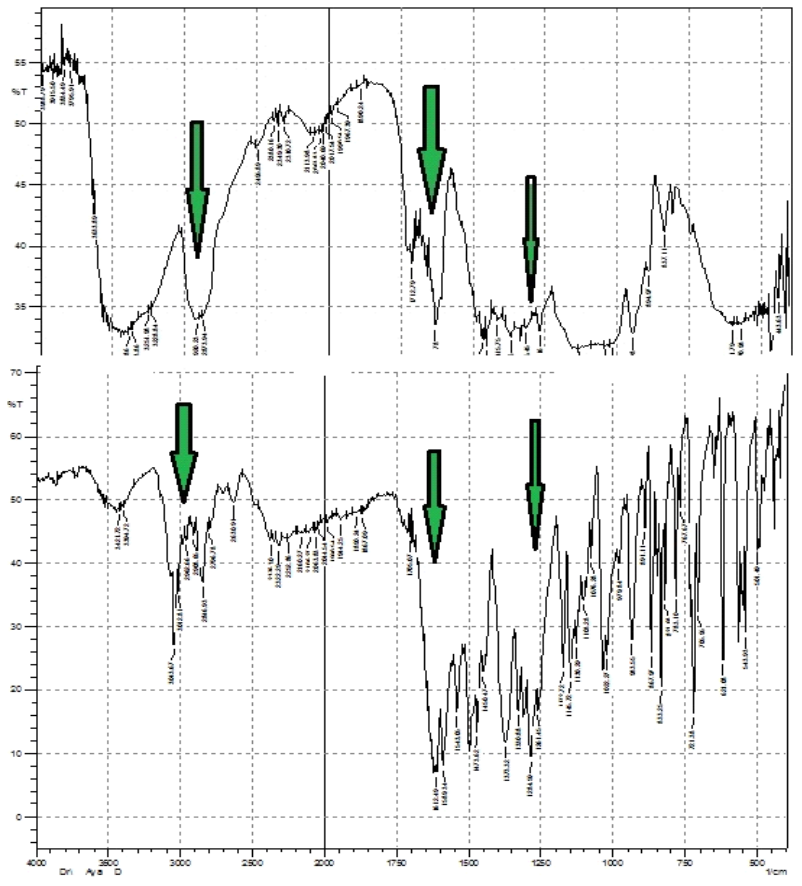

Fig. 2: FT-IR spectra of the drug-loaded ocular insert of HPMC (a) and the CFX $\mathrm{HCl}$ (b)

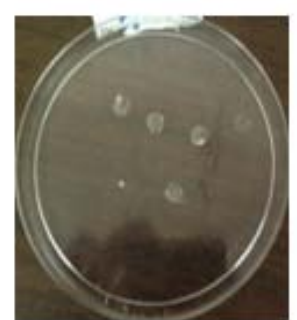

III

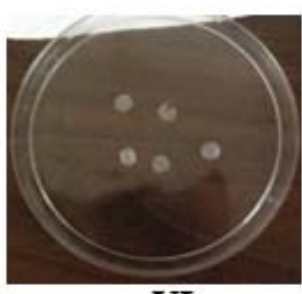

VI

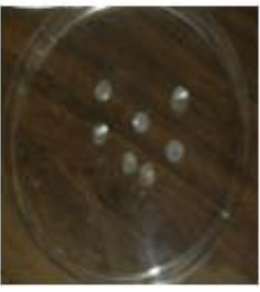

VII

Fig. 3: Photos representing the fabrication of ocuserts

\section{Characterization of the prepared ocuserts}

The prepared ocuserts were evaluated for their thickness at three random points. All ocuserts showed uniformity in thickness as indicated by low SD of the measured thickness. Variations in the ocusert thickness is reflected by the different amount of polymer needed for ocusert fabrication (table 2).

Uniformity of the weight of the ocuserts is illustrated by low SD obtained after measurement. The mean weight value varied between $2.44 \pm 0.15 \mathrm{mg}$ to $8.4 \pm 1.3 \mathrm{mg}$, as it's affected proportionally by the ocusert thickness.

The mean values of three replicates of both \% moisture loss and \% moisture gain were recorded in table 2. Formulae F-V and F-VI become brittle upon exposure to moisture loss while formulae III and IV showed gelation of the film and in formula $\mathrm{V}$ disintegration of the film into fragments took place upon moisture gain. These observations are the net result of different hydration properties of different polymers.

All prepared formulae showed uniform drug content in the range of $94.14 \pm 8.22 \%$ to $98.22 \pm 5.02 \%$ with low SD values.

Folding endurance of all batches was found between $19 \pm 6$ to $72 \pm 11$, the significant difference in the folding endurance of the prepared films is due to the use of different polymer types and concentrations.

The values of surface $\mathrm{pH}$ vary between 6.2 to 7.2 which give rise to the assumption that the ocuserts will cause no irritation upon insertion in the eye. 
Table 2: Physicochemical evaluation of different formulations*

\begin{tabular}{|c|c|c|c|c|c|c|c|}
\hline Formulae & $\begin{array}{l}\text { Thickness } \\
\text { (mm) }\end{array}$ & $\begin{array}{l}\text { Weight } \\
\text { (mg) }\end{array}$ & Moisture loss \% & Moisture gain \% & Drug content \% & Folding endurance & Surface pH \\
\hline F-I & $0.263 \pm 0.05$ & $4.6 \pm 0.24$ & $21.6 \pm 7.4$ & $36.4 \pm 7.8$ & $94.6 \pm 7.4$ & $56 \pm 7$ & 6.23 \\
\hline F-II & $0.34 \pm 0.02$ & $8.4 \pm 1.3$ & $24.8 \pm 6.6$ & $33.2 \pm 11.4$ & $97.04 \pm 4.6$ & $72 \pm 11$ & 6.2 \\
\hline F-III & $0.24 \pm 0.07$ & $4.6 \pm 0.07$ & $6.8 \pm 2.7$ & Gelation & $98.22 \pm 5.02$ & $45 \pm 6$ & 6.22 \\
\hline F-IV & $0.22 \pm 0.00$ & $4.9 \pm 0.63$ & $12.8 \pm 3.3$ & Gelation & $95.7 \pm 12.7$ & $24 \pm 4$ & 7.1 \\
\hline F-V & $0.31 \pm 0.04$ & $5.7 \pm 0.05$ & Brittle & Disintegrated & $98.02 \pm 9.7$ & $22 \pm 6$ & 6.66 \\
\hline F-VI & $0.32 \pm 0.02$ & $6.42 \pm 0.26$ & $30.5 \pm 8.5$ & $34.7 \pm 13.8$ & $96.37 \pm 10.2$ & $62 \pm 14$ & 6.8 \\
\hline F-VII & $0.316 \pm 0.04$ & $2.44 \pm 0.15$ & Brittle & $35.4 \pm 11.2$ & $94.14 \pm 8.22$ & $19 \pm 8$ & 7.2 \\
\hline
\end{tabular}

* (Results are expressed as mean \pm SD, $n=3$ )

\section{Bioadhesion characterization}

\section{Ex vivo bioadhesion strength}

Bioadhesion may be defined as the adhesion between a polymer and a biological membrane, e. g. mucus. The strength of bio-adhesion is affected by numerous factors such as the molecular weight of polymers, contact time with mucus, swelling rate of the polymer, and biological membrane used in the study. All ocuserts showed appreciable bioadhesive detachment stress that ranged between $240 \pm 66$ and $158 \pm 52$ dyne $/ \mathrm{cm}^{2}$ (fig. 4) indicating a potential of sustaining the stay and enhancing contact with cornea. Various mechanisms have been proposed to explain the in vitro bioadhesion or mucoadhesion phenomena such as electrical double layers, electrostatic attractions, hydrogen bonding, Van der Waals force, hydrophobic bonding, wetting, diffusion-interpenetration, physical entanglements, and surface-free energy [40].

Most of the hydrophilic polymers can absorb water and swell. This can increase the potential to adhere onto mucosal surfaces. This is the simplest mechanism of adhesion and has been defined as "adhesion by hydration".

Na CMC can increase surface charge density, and the carboxylic group can form hydrogen bonds with tissue [40]. HPMC is the long chained, non-ionic polymer and the mucoadhesive property could be due to the formation of physical or hydrogen bonding with the mucus components. HPMC can relieve the dryness and irritation even in the case of reduced mucus secretions [40, 41]. Na CMC and HPMC show faster hydration rate and thereby swelling which helps in the interpenetration of mucus and polymer resulting in bio-adhesion.

HPMC is a non-ionic polymer containing only hydroxyl groups, which can form weak hydrogen bonds with mucous layers. Furthermore, owing to its slow rate of hydration it can form a strong surface gel that efficiently adheres onto the mucosal surface and remains in contact for a longer time. For this reason, it can be characterized as one of the most effective mucoadhesive polymers $[25,42]$.

The highest bioadhesive force showed by F-IV containing HPC while the lowest value showed by F-VI containing pullulan.

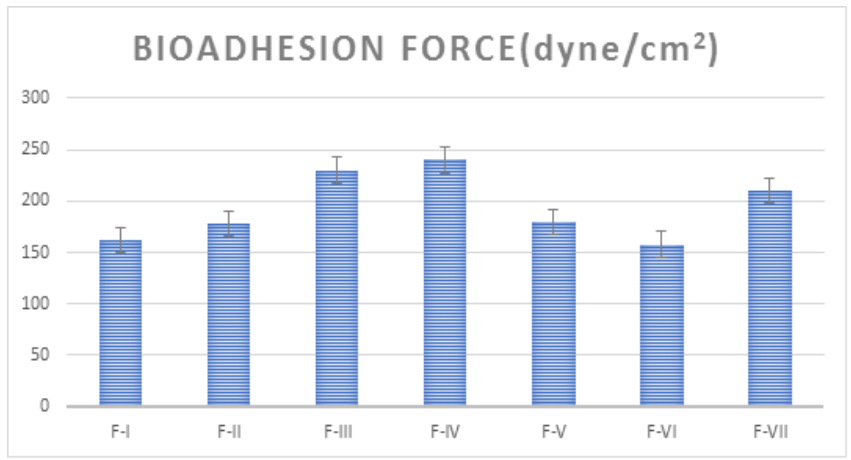

Fig. 4: Histogram representing the bioadhesion force of different ocuserts, (Results are expressed as mean $\pm S D, n=3$ )

\section{Ex vivo bioadhesion time}

The ex vivo bioadhesion time (residence time) of ocuserts varied from $2360 \pm 140$ to $260 \pm 32$ s. (fig. 5). It was observed that a gradual increase in the residence time occurred with a concomitant increase in the polymer viscosity. The observation can be assigned to the inherent property of the polymer HPMC that although showing significantly higher swelling is less water affined and hence tends to retain its structure better. In addition, increased viscosity led to the formation of a surface gel that maintained its structural integrity for a longer period of time, thereby resulting in increased residence time.

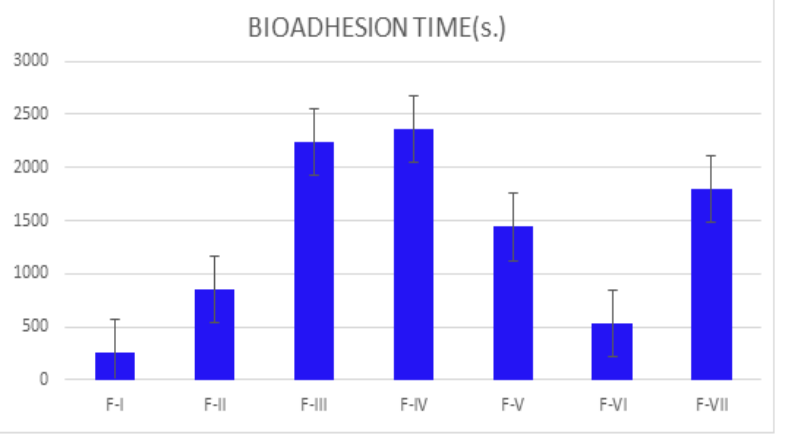

Fig. 5: Histogram representing the bio-adhesion time of different ocuserts. (Results are expressed as mean $\pm S D, n=3$ )

\section{In vitro drug release study}

The cumulative percent of $\mathrm{CFX} \mathrm{HCl}$ released from ocuserts as a function of time is shown in Fig. 6 which revealed that $99 \%$ of the drug was released from HPMC based ocuserts in about $5 \mathrm{~h}$.

$\mathrm{Na}$ Alg. and pullulan based ocuserts showed very close results (i.e. $\mathrm{Na}$ Alg. based ocuserts released about $94 \%$ and pullulan based ocuserts released about $92 \%$ in $8 \mathrm{~h}$ ).

PVA, HPC and XG based ocuserts released 98\%, 92\% and 90\% respectively in about $24 \mathrm{~h}$. The most sustaining effect is attained by $\mathrm{Na}$ CMC based ocuserts where it shows only the release of $34 \%$ in about $24 \mathrm{~h}$

The enormous difference in drug release may be explained by the change in the polymer forming the ocuserts. Generally, drug release from the polymeric matrices is elicited by the ease of water accessibility into the matrix, which breaks the polymer-polymer bonds and thus simultaneously leads to bounding of water and polymer molecules, separation of polymer chains, swelling to form a gel, and finally dispersion of polymer chains in the medium. "The drug dissolves in the gel and diffuses to the exterior with a rate depending on its concentration gradients and its diffusion ability through the gel. Concurrently, the latter is eroded with a rate depending on polymer molecular weight and hydrodynamics of release medium. The drug release pattern depends on the relative rates of these processes" [43]. 
As, HPMC is a hydrophilic polymer which facilitates ease of water penetration into the polymer matrix and hence the ease of drug diffusion. Although pullulan is also hydrophilic polymer composed of polysaccharide, it shows more sustaining in drug release than HPMC due to a greater amount of pullulan is needed in fabricating satisfactory films other than HPMC where a lesser amount is sufficient in fabricating film with satisfactory mechanical strength.

$\mathrm{Na}$ Alg. showed more retardation in drug release reached to $8 \mathrm{~h}$ to complete release of the incorporated drug. This may be explained by features of Na Alg. in which hydrophilic functional groups of the polymer and water molecules tend to bound together by hydrogen bonding leading to excessive swelling results in retarding of drug release [44].

In case of PVA (a hydrophilic polymer); but in its processing in formulating a film, it was heated to $70{ }^{\circ} \mathrm{C}$ and plasticized by PG. Both heat and PG addition caused crosslinking of PVA resulting in retardation in drug release [45].

The release pattern of HPC can be explained by high swelling behavior which results into inclusion of water inside the polymer matrix resulting into retardation in drug release [46].

$\mathrm{XG}$ is considered a good matrix-forming material for sustainedrelease tablets as it tends to form viscous gels in the presence of water, whether they are used alone or in combination with other gums or polymers. This property also affects the drug release from ocuserts as seen in the dissolution behaviour [46].

In case of Na CMC there was great retardation in drug release although its hydrophilic character it had great swelling behaviour and inclusion of water molecules into the polymer matrix results in the formation of gel into the polymer matrix causes retardation in drug release as seen the remaining of gel in the diffusion cell at the end of $24 \mathrm{~h}$ but it may be promising in preparing formulations for several days but in ocular route it may be annoying to the patient so its excluded from further study.

By overviewing the aforementioned release pattern, F-II was selected as a promising ocuserts providing the overall incorporated drug in $24 \mathrm{~h}$ comply with the hypothesis of the study by formulating once a day dose.

Considering the drug release kinetics when the data was plotted as cumulative $\%$ of the drug released vs. time according to zero order equation, F. I, VI and VII showed a fair linearity indicting higher correlation than first order and Higuchi equation, with the highest $\mathrm{R}^{2}$ values compared to other plots, while, F. II, III, and IV showed a fair linearity with first order indicting higher correlation than zero order and Higuchi equation, with the highest $\mathrm{R}^{2}$ values compared to other plots except for Alginate showing diffusion release. The zero-order kinetics [47] reveals delivery of the drug in a sustained manner whereby the drug is held in a reservoir representing the ocusert and is released at a constant rate to provide a constant concentration in the cornea which provides improved patient compliance $[48,49]$.

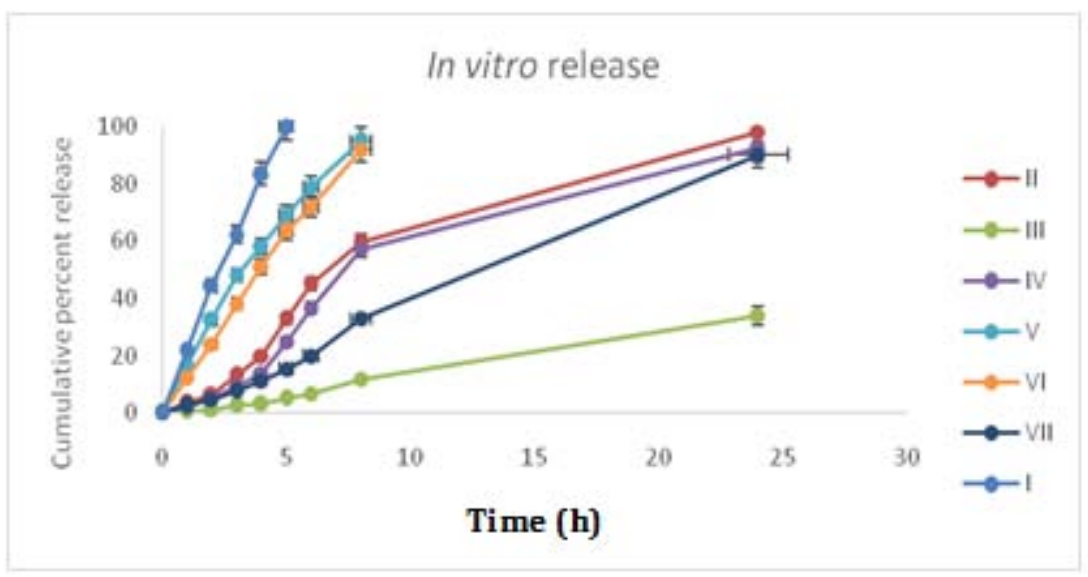

Fig. 6: In vitro cumulative percent release of $\mathrm{CFX} \mathrm{HCl}$ ocuserts, (results are expressed as mean $\pm \mathrm{SD}, \mathrm{n}=3$ )

Table 3: $\mathbf{R}^{2}$ correlation values of different kinetic models of CFX HCl ocuserts

\begin{tabular}{llll}
\hline Formula & $\mathbf{R}^{\mathbf{2}}$ of zero & $\mathbf{R}^{\mathbf{2}}$ of first & $\mathbf{R}^{\mathbf{2}}$ of higuchi \\
\hline F-I & 0.998568 & 0.82807 & 0.996927 \\
F-II & 0.940915 & 0.99503 & 0.978563 \\
F-III & 0.997076 & 0.99716 & 0.973945 \\
F-IV & 0.943501 & 0.99489 & 0.972235 \\
F-V & 0.990315 & 0.96156 & 0.999468 \\
F-VI & 0.996656 & 0.96004 & 0.995587 \\
F-VII & 0.99752 & 0.98684 & 0.97749 \\
\hline
\end{tabular}

\section{Stability studies}

Formula F-II was subjected to accelerated stability study to determine the physical stability of the formulation. There were no significant changes regarding the physical properties at the end of the three months and drug content during the study period. The overall degradation is less than $1.25 \%$. A tentative shelf-life of one year may be assigned to formulation as per ICH guidelines. No change in physical appearance of ocusert was reported during the period of study thus revealing that F II passed the stability test indicating that it was chemically, physically and microbiologically stable at the examined temperature for 3 mo. However, its shelf life needs to be established by further studies at different temperatures and humidity conditions.

\section{UV irradiation}

The efficiency of the sterilization process is indicated mainly by sterility test where the ocuserts is examined for sterility by two media where it showed no sign of turbidity after $7 \mathrm{~d}$ for Medium $\mathrm{A}$ (fluid thioglycollate medium) and Medium B (soyabean-casein digest medium) indicating excellent sterility of the tested ocuserts.

\section{In vivo drug release and irritation study}

The results of in vivo drug release study are presented in fig. 7. The ocusert for the first $5 \mathrm{~h}$ can be removed from the rabbit eye and tested for drug remaining. After the first $5 \mathrm{~h}$ the ocusert adhesive to the ocular surface and cannot be removed. The rabbits also examined for eye irritancy for $7 \mathrm{~d}$ after the study and no rabbit show any sign of irritancy 
(score 0) for all formulations. The in vivo drug release study from F. II was found to be in accordance with that of the in vitro drug release study. Hence, we tried to correlate in vivo results with the in vitro percentage drug release. The correlation value was found to be 0.9982 [50]
Therefore, the formula (F-II) exhibited strong in vitro-in vivo correlation revealing the efficacy of the formulation (fig. 8). No drag out of circular inserts at the time of experiment was happened which suggest that the dimension ( $8 \mathrm{~mm})$ was suitable as ocuserts [50].

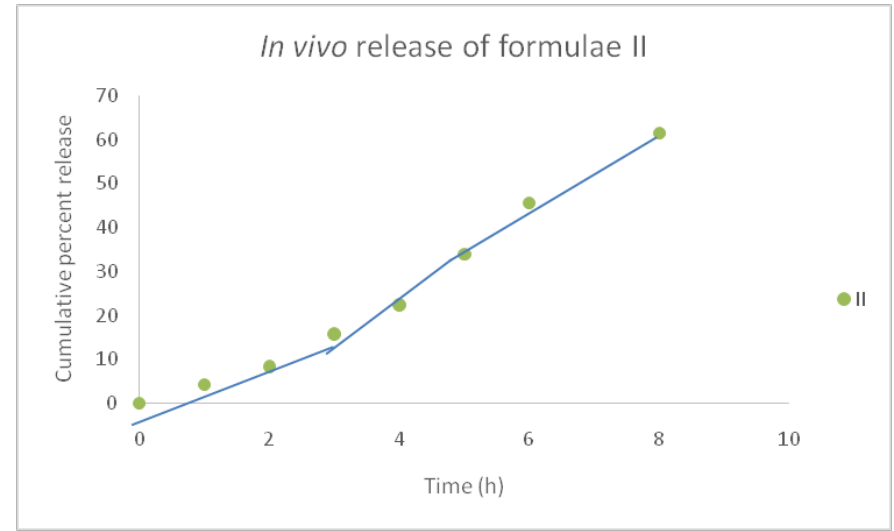

Fig. 7: In vivo cumulative percent release of formula II, (results are expressed as mean $\pm \mathrm{SD}, \mathrm{n}=3$ )

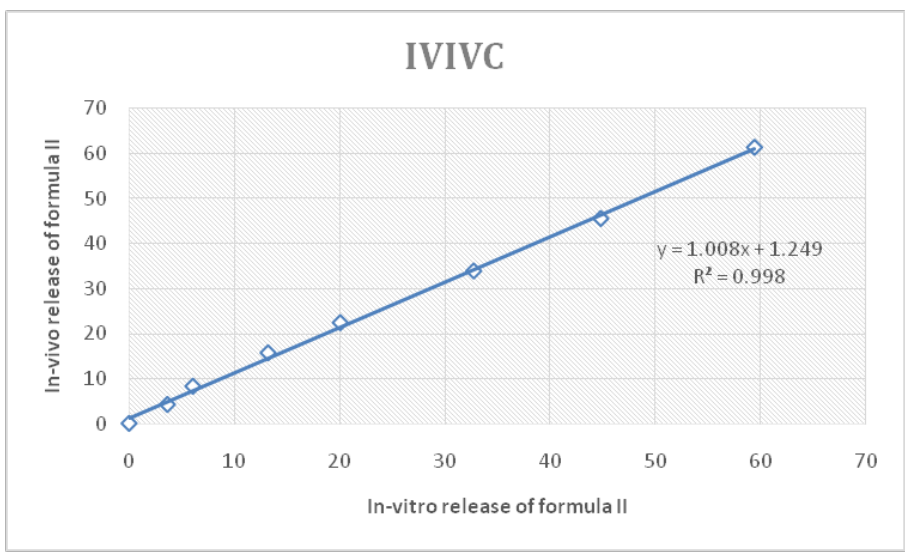

Fig. 8: In vitro In vivo correlation of formula II, (results are expressed as mean \pm SD, $n=3$ )

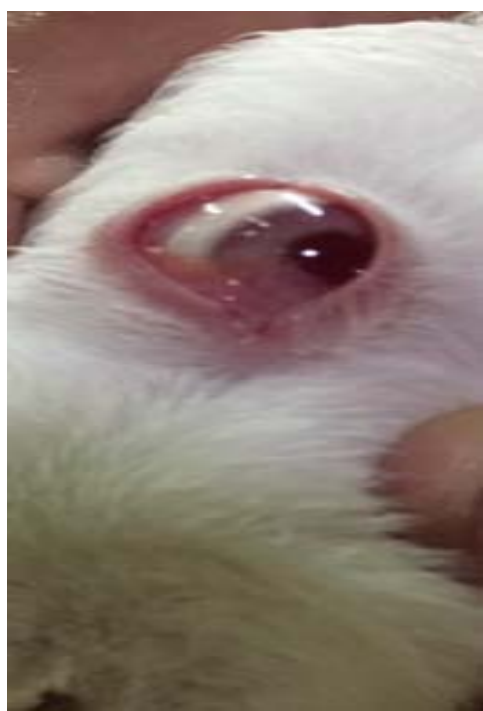

(a)

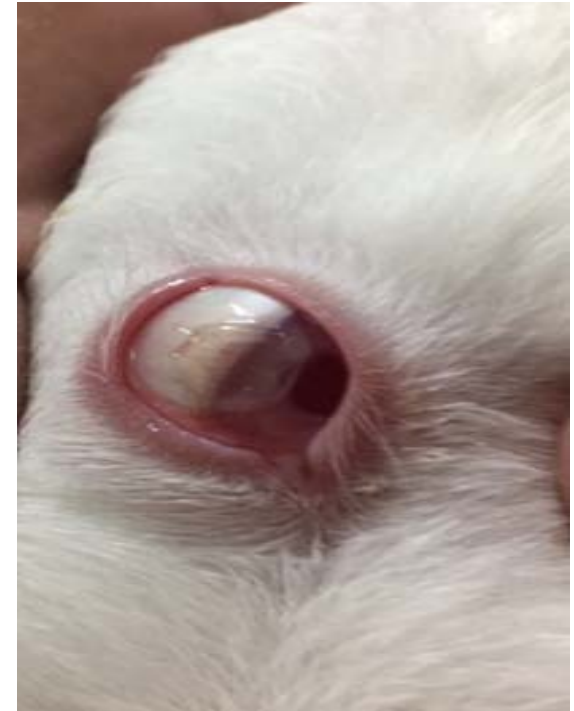

(b)

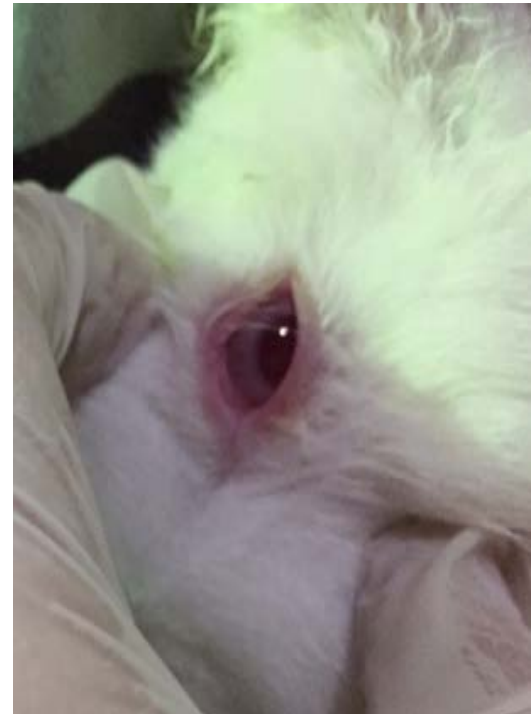

(c)

Fig. 9: Sequence of photos of in vivo experiment showing (a) before insertion of ocusert (b) during removal of ocusert (c) and after the complete dissolving of ocusert after $24 \mathrm{~h}$ 


\section{Microbiological studies}

Testing the selected ocuserts (Formulae F-II and F-III) against staph aureus showed good antimicrobial activity indicted by clear zones of inhibitions (3 cm diameter for each formulae) when tested microbiologically on solidified agar. While control ocusert (ocusert with no drug) shows no zone of inhibition as represented in fig. 10 [51].

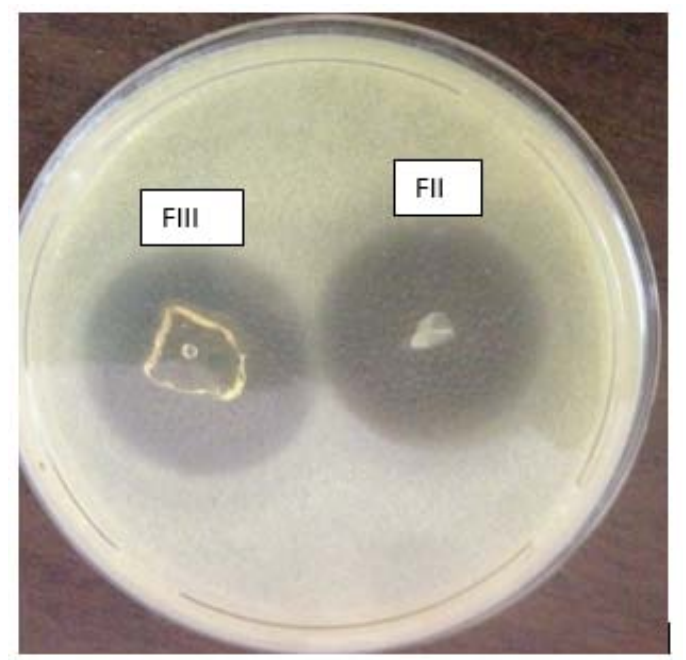

Fig. 10: Photo represents the microbiological study of formula II and III ocusert against S. Aureus

\section{CONCLUSION}

Various bioadhesive formulations of $\mathrm{C} \mathrm{F} \mathrm{X} \mathrm{HCl}$ ocuserts were prepared using a solvent casting method and evaluated. Ocuserts (formula F-II) consisting of a 1.5\% PVA with $2.5 \%$ PG satisfied all the pharmaceutical parameters of bioadhesive ocuserts and demonstrated controlled release of the drug in the eye over the period of $24 \mathrm{~h}$. Formula F-II is considered a promising formulation suitable for a once a day dose and thereby improving the patient compliance by providing benefits of reducing in the frequency of administration by controlled drug release. Pharmacokinetics and pharmacodynamics studies in human beings are needed to be carried out to establish the therapeutic utility of this system.

\section{ACKNOWLEDGMENT}

Authors are thankful to Dr. Nagwan Glal, Lecturer of Microbiology, Microbiology Department, Faculty of Pharmacy Al-Azhar University for her grateful assistance in conducting microbiological studies.

\section{AUTHORS CONTRIBUTIONS}

All the author have contributed equally

\section{CONFLICT OF INTERESTS}

No conflicts of interest were reported by the authors. Only the authors are responsible for the scientific hypothesis, performance of the work and writing of this paper.

This research did not receive any specific grant from funding agencies in the public, commercial, or not-for-profit sectors.

\section{REFERENCES}

1. Peneva PT. Non-steroidal anti-inflammatory drugs for topical ophthalmic administration: contemporary trends. Int J Pharm Pharm Sci 2015;7:13-9.

2. Sharma PK, Kumar A, Bajpai M, Sachdeva M, Kumar P. Formulation, optimization and evaluation of ocular inserts of ciprofloxacin hydrochloride. Biosci Biotechnol Res Asia 2008;5:757-62.

3. Alany RG, Rades T, Nicoll J, Tucker IG, Davies NM. W/O microemulsions for ocular delivery: Evaluation of ocular irritation and precorneal retention. J Controlled Release 2006;111:145-52.

4. Nanjawade BK, Manvi FV, Manjappa AS. In situ-forming hydrogels for sustained ophthalmic drug delivery. J Controlled Release 2007;122:119-34.

5. Sahoo KS, Dilnawaz F, Krishnakumar S. Nanotechnology in ocular drug delivery. Drug Discovery Today 2008;13:144-51.

6. Andrews GP, Laverty TP, Jones DS. Mucoadhesive polymeric platforms for controlled drug delivery. Eur J Pharm Biopharm 2009;71:505-18.

7. Amar A, Ashish K, Ajaykumar P, Anand J. Formulation and evaluation of controlled release ocuserts of betaxolol hydrochloride. IOSR J Pharm 2012;2:34-8.

8. Abdou EM, Kandil SM. Formulation and evaluation of dorzolamide and timolol ocuserts. Int J Pharm Sci Res 2017;8:915-24.

9. Tofighia P, Soltani S, Montazam SH, Montazam SA, Jelvehgari M. formulation of tolmetin ocuserts as carriers for ocular drug delivery system. Iran J Pharm Res 2017;16:432-41.

10. Chourasia A, Agrawal S. Development and evaluation of ciprofloxacin hydrochloride loaded ocular insert by using "PLANTAGO OVATA" as a natural polymer. Int J Curr Pharm Res 2018;10:79-88.

11. Shah HP, Prajapati ST, Patel CN. Gastroretentive drug delivery systems: from conception to commercial success. J Crit Rev 2017;4:10-21.

12. Kharenko EA, Larionova NI, Demina NB. Mucoadhesive drug delivery systems (Review). Pharm Chem J 2009;43:200-8.

13. Koelwel C, Rothschenk S, Fuchs Koelwel B, Gabler B, Lohmann C, Gopferich A. Alginate inserts loaded with epidermal growth factor for the treatment of keratoconjunctivitis sicca. Pharm Dev Technol 2008;13:221-31.

14. Deshpande PB, Dandagi P, Udupa N, Gopal SV, Jain SS, Vasanth SG. Controlled release polymeric ocular delivery of acyclovir. Pharm Dev Technol 2010;15:369-78.

15. Blondeau JM. Fluoroquinolones: mechanism of action, classification, and development of resistance. Surv Ophthalmol 2004;49:S73-S8.

16. Shazly GA. Ciprofloxacin controlled-solid lipid nanoparticles: characterization, in vitro release, and antibacterial activity assessment. Biomed Res Int 2017:1-9. Doi:10.1155/2017/ 2120734.

17. Abhilash AS, Jayaprakash S, Nagarjan M, Dhachinamoorthi D. Design and evaluation of timolol ocuserts. Indian J Pharm Sci 2005;67:311-4.

18. Nayak BS, Patnaik SC, Sethy S, Ellaiah P, Sarangi BK. Formulation design and in vitro-in vivo evaluation of moxifloxacin ophthalmic insert. Am J Phytomed Clin Ther 2015;3:302-12.

19. Abul Ella SS. A pharmaceutical study on an antihistaminic drug absorbed from the buccal cavity", [MSc Thesis], Misr university for science and technology (MUST). $6^{\text {th }}$ of October governorate, Egypt; 2011.

20. Balasubramaniam J, Srinatha A, Pandit JK. In vitro microbiological evaluation of polyvinyl alcohol-based ocular inserts of ciprofloxacin hydrochloride. Indian J Pharm Sci 2006;68:626-30.

21. Kaul S, Kumar G, Kothiya P. Design and evaluation of soluble ocular drug insert for controlled release of acyclovir. Int J Drug Res Tech 2012;2:393-8.

22. Sharma J, Sharma S, Kaura A, Durani S. Formulation and in vitro evaluation of moxifloxacin ocular inserts. Int J Pharm Pharm Sci 2014;6:177-9.

23. Ale A, Sharma SN. Fabrication of a through-flow apparatus for in vitro determination of drug from ophthalmic preparations. Ind Drugs 1991;29:157-60.

24. Ashture A, Kankudte A, Patil A, Joshi A. Formulation and evaluation of controlled release ocular inserts of betaxolol hydrochloride. IORS J Pharm 2012;2:34-8.

25. Gilhotra RM, Gilhotra N, Mishra DN. Piroxicam bioadhesive ocular inserts: physicochemical characterization and evaluation in prostaglandin-induced inflammation. Curr Eye Res 2009;34:1065-73.

26. Ikram M, Gilhotra N, Gilhotra RM. Formulation and optimization of mucoadhesive buccal patches of losaratan 
potassium by using response surface methodology. Adv Biomed Res 2015;4:239.

27. Patel VM, Prajapati BG, Patel MM. Design and characterization of chitosan-containing mucoadhesive buccal patches of propranolol hydrochloride. Acta Pharm 2007;57:61-72.

28. Higuchi T. Mechanism of rate of sustained-action medication. J Pharm Sci 1963;52:1145-9.

29. Grattarda N, Perninb M, Martyb B, Roudauta G, Champion D. Study of the release kinetics of small and high molecular weight substances dispersed into spray-dried ethylcellulose microspheres. J Controlled Release 2002;84:125-35.

30. Dave V, Paliwal S, Yadav S. Formulation and evaluation of controlled delivery of aceclofenac through the ocular insert. Turk J Pharm Sci 2013;10:205-20.

31. Guidance for Industry Q1A (R2) Stability testing of new drug substances and products; 2003.

32. Al shawwa SZ, Obaidat A, Sallam A, Salem MS. In vivo study of promising formulated ocular bio-adhesive inserts of ciprofloxacin hydrochloride combination with xanthan gum and carbopol. Int J Pharm Pharm Sci 2017;9:229-5.

33. Abdelkader H, Piercionek B, Alany RG. Novel in situ gelling ocular films for the opioid growth factor-receptor antagonistnaltrexone hydrochloride: Fabrication, mechanical properties, mucoadhesion, tolerability and stability studies. Int J Pharm 2014;477:631-42.

34. Peyman GA, Paque JT, Meisels HI, Bennett TO. Postoperative endophthalmitis: a comparison of methods for treatment and prophlaxis with gentamicin. Ophthalmic Surg 1975;6:45-55.

35. Pawar PK, Katara R, Majumdar DK. Design and evaluation of moxifloxacin hydrochloride ocular inserts. Acta Pharm 2012;62:93-104.

36. Sankar V, Chandrasekaran AK, Durga S, Geetha G, Ravichandran VA. Design and evaluation of diclofenac sodium ophthalmic inserts. Acta Pharm Sci 2006;48:5-10.

37. Shanmugam S, Valarmathi S, Satheesh K. Sterility testing of procedure of ophthalmic ocuserts acyclovir used for treating herpes simplex virus. Asian J Pharm Clin Res 2017;10:344-6.

38. Sarath CC, Shirwalkar A, Kiron SS. Development and evaluation of chitosan containing ciprofloxacin $\beta$-CD complex. Int J Pharm Tech Res 2010;2:246-52.
39. Attia MA, Al-Azizi M, Hashish MS. Design and evaluation of ciprofloxacin hydrochloride ocular inserts. Int J Pharm Tech Res 2011;3:1750-63.

40. Sudeendra BR, Umme H, Gupta RK, Shivakumar HG. Development and characterization of bioadhesive vaginal films of clotrimazole for vaginal candidiasis. Acta Pharm Sci 2010;52:417-26.

41. Yoo JW, Dharmala K, Lee CH. The physicodynamic properties of mucoadhesive polymeric films developed as female controlled drug delivery system. Int J Pharm 2006;309:139-45.

42. Karavas E, Georgarakis E, Bikiaris D. Application of PVP/HPMC miscible blends with enhanced mucoadhesive properties for adjusting drug release in predictable pulsatile chronotherapeutics. Eur J Pharm Biopharm 2006;64:115-26.

43. Joshi HN, Wilson TD. Calorimetric studies of dissolution of hydroxypropyl methylcellulose E5 (HPMC E5) in water. J Pharm Sci 1993;82:1033-8.

44. Wu $\mathrm{K}, \mathrm{XU} \mathrm{ZL}$, Wei YM. Sodium alginate-polyvinyl alcohol/polysulfone (SA-PVA/PSF) hollow fiber composite pervaporation membrane for dehydration of ethanol-water solution. J Shanghai Univ (English Ed) 2008;12:163-70.

45. Kodavaty J, Deshpande AP. Mechanical and swelling properties of poly (vinyl alcohol) and hyaluronic acid gels used in biomaterial systems a comparative study. Def Sci J 2014;64:222-9.

46. Siepmann J, Siegel RA, Rathbone MJ. Fundamentals and applications of controlled release drug delivery. Springer: NY, USA; 2012. p. 493-516.

47. Indian Pharmacopoeia. Vol. I and II; 1996.

48. Manjula BS, Srinatha A, Sridhar BK. Evaluation of hydrophilic polymers and their combinations in the formulation of sustained-release matrix tablets of a water-soluble drug. Indian J Pharm Edu Res 2014;48:48-69.

49. Nithiyananthan TS, Shankarananth V, Rajasekhar KK, Jyothikrishna $\mathrm{K}$, Mukesh O, Vikram KE. Preparation and evaluation of ciprofloxacin ocuserts. J Pharm Res 2009;2:1496-9.

50. Sreenivas SA, Hiremanth SP, Godbole AM. Ofloxacin ocular inserts: design, formulation, and evaluation. Iran J Pharmacol Ther 2006;5:159-62.

51. Mundada A, Shrikhande BK. Formulation and evaluation of ciprofloxacin hydrochloride soluble ocular insert. Curr Eye Res 2008;33:469-75. 